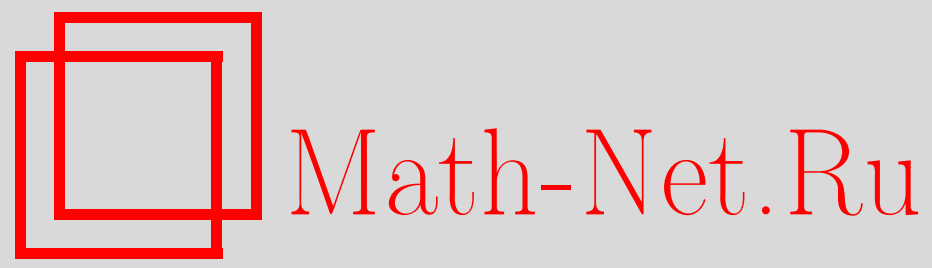

Ж. Муссафир, Паруса и базисы Гильберта, Функи. анализ и его прил., 2000, том 34, выпуск 2, 43-49

DOI: https://doi.org/10.4213/faa294

Использование Общероссийского математического портала MathNet.Ru подразумевает, что вы прочитали и согласны с пользовательским соглашением http://www.mathnet.ru/rus/agreement

Параметры загрузки:

IP: 54.237 .206 .68

26 апреля 2023 г., 12:38:31

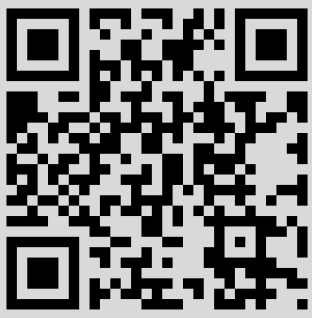


Функциональньй анализ и его приложения

2000, т. 34, вып. 2, с. 43-49

УДК $512.7+514.17$

\section{Паруса и базисы Гильберта}

(с) 2000. Ж.-О. МУССАФИР

\section{Введение}

Полиэдр Клейна - это выпуклая оболочка ненулевых целых точек симплициального конуса $C \subset \mathbb{R}^{n}$. Имеется связь между этими полиэдрами и базисами Гильберта полугрупп целых точек, содержащихся в симплициальном конусе.

В двумерном случае множество целых точек, лежащих на границе многоугольника Клейна, содержит базис Гильберта соответствующей полугруппы. Если размерность больше либо равна 3, это уже, вообще говоря, не так (см., например, [2]). Но мы дадим в трехмерном случае полное описание полиэдров, обладающих этим свойством. Мы приведем пример такого паруса и покажем, что наш критерий не выполняется в размерности 4.

\section{$\S 1$. Базисы Гильберта}

Приведем некоторые определения (полное изложение см. в [4]).

Пусть векторы $a_{1}, \ldots, a_{n} \in \mathbb{R}^{n}$ удовлетворяют условию $\operatorname{det}\left(a_{1}, \ldots, a_{n}\right) \neq 0$. Определим векторы двойственного базиса $w_{1}, \ldots, w_{n}$ условием $\left\langle a_{i}, w_{j}\right\rangle=\delta_{i j}$ для $i, j=1, \ldots, n$, где $\delta_{i j}$ - символ Кронекера. Симплициальный конус, порожденный векторами $a_{1}, \ldots, a_{n}$, 一 это множество

$$
C=\left\{\lambda_{1} a_{1}+\cdots+\lambda_{n} a_{n}, \text { где } \lambda_{i} \in \mathbb{R}^{+}\right\} .
$$

Разумеется,

$$
C=\left\{x \in \mathbb{R}^{n} \mid\left\langle w_{i}, x\right\rangle \geqslant 0 \text { при } i=1, \ldots, n\right\} .
$$

Пусть $C$ - симплициальный конус. Определим полугруппу $M$ формулой $M=C \cap \mathbb{Z}^{n}$, и пусть $K$ - выпуклая оболочка множества $M^{*}=M \backslash\{O\}$. Парус $V$ - это граница множества $K$, а $E=\bigcup_{x \in M^{*}} C+x$. Будем называть конус $C$ рациональным, если $a_{i} \in \mathbb{Z}$ при $i=1, \ldots, n$. В этом случае также будем говорить, что соответствующий парус рационален. Некоторые грани паруса не содержат начала координат. Объединение этих граней называется приведенным парусом и обозначается через $\widetilde{V}$.

Скажем, что аффинная гиперплоскость в $\mathbb{R}^{n}$ является целочисленной, если она содержит $(n-1)$-мерную целочисленную решетку. Мы будем также говорить о целочисленных прямых.

ОПРеДЕЛЕНИЕ 1 . Пусть $C$ - симплициальный конус, а $M-$ полугруппа его целых точек. Базис Гильберта полугруппы $M$ - это минимальное множество образующих этой полугруппы.

Существование такого минимального множества вытекает из леммы Цорна. Нетрудно видеть, что множество $\widetilde{V} \cap \mathbb{Z}^{n}$ содержится в каждом базисе Гильберта полугруппы $M$. В самом деле, пусть $B$ - базис Гильберта для $M$ и $x \in \widetilde{V} \cap \mathbb{Z}^{n}$. 
Пусть $F$ - грань, содержащая $x$, а $H$ - гиперплоскость, содержащая $F$. Гиперплоскость $H$ задается рациональным уравнением $v(x)=d$, где $d \in \mathbb{N}^{*}$, и отделяет $O$ от $M^{*}: v(y) \geqslant d$ для всех $y$ из $M^{*}$. Таким образом, если

TO

$$
x=a_{1} x_{1}+\cdots+a_{k} x_{k}, \quad a_{i} \in \mathbb{N}, x_{i} \in B,
$$

$$
v(x)=d \geqslant\left(a_{1}+\cdots+a_{k}\right) d .
$$

Все $a_{i}$, кроме одного, равны нулю, и, следовательно, $x \in B$.

Следующая теорема является адаптацией результата из [2].

Teорема 1. Существует единственное множество $S \subset M^{*}$, такое, что

1) $E=\bigcup_{x \in S} C+x$

2) если $x, y \in S, x \neq y$, mo $C+x \not \subset C+y$ u $C+y \not \subset C+x$.

ДокАЗАТЕЛЬСтво. УПорядочим множества $x+C, x \in M^{*}$, по включению. Положим

$$
S=\left\{x \in M^{*} \mid C+x \text { максимально }\right\} .
$$

Пусть $x \in M^{*}$. Если $C+x$ не содержится в $\bigcup_{x \in S} C+x$, то $x$ не принадлежит $S$, $C+x$ не максимально и существует точка $x_{1} \in M^{*}$, такая, что $C+x \subsetneq C+x_{1}$. Если бы точка $x_{1}$ лежала в $S$, мы бы имели $C+x \subsetneq C+x_{1} \subset \bigcup_{x \in S} C+x$. Таким образом, $x_{1} \notin S$. Построим последовательность $x_{0}=x, x_{1}, x_{2}, \ldots$, такую, что $C+x_{0} \subsetneq C+x_{1} \subsetneq C+x_{2} \subsetneq \ldots$

Нетрудно проверить, что $\left(\left\langle w_{i}, x_{k}\right\rangle\right)_{k \in \mathbb{N}}$ - убывающие последовательности положительных чисел и что $\left(x_{k}\right)_{k \in \mathbb{N}}$ сходится в $\mathbb{R}^{n}$. Но поскольку $M^{*} \subset \mathbb{Z}^{n}$, эта последовательность должна быть постоянной, начиная с некоторого места, что противоречит условию $C+x_{k} \subsetneq C+x_{k+1}$ для любого $k$. Отсюда получаем, что $E=\bigcup_{x \in S} C+x$. Множество $S$ удовлетворяет также условию 2 ).

Сейчас мы должны показать, что $S$ единственно. Пусть $S^{\prime} \in M^{*}$ удовлетворяет условиям 1) и 2). Если $x \in S$, то существует элемент $x^{\prime} \in S^{\prime}$, такой, что $x \in C+x^{\prime}$ и $C+x \subset C+x^{\prime}$. Но $C+x$ максимально; поэтому $x=x^{\prime}$. Таким образом, $S \subset S^{\prime}$. Обратно, если $x \in S^{\prime}$ и $C+x$ не максимально, то найдется $y \in M^{*}$, такой, что $C+x \subset C+y$. Но поскольку $E=\bigcup_{x^{\prime} \in S^{\prime}} C+x^{\prime}$, существует $x^{\prime} \in S^{\prime}$, такой, что $C+y \subset C+x^{\prime}$. Мы нашли элементы $x$ и $x^{\prime}$ в $S^{\prime}$, такие, что $x \neq x^{\prime}$ и $C+x \subset C+x^{\prime}$. Это противоречит условию 2$)$, так что $C+x$ максимально и $S^{\prime} \subset S$.

Множество $S$, которое мы только что построили, является базисом Гильберта полугруппы $M$. Для того чтобы это доказать, нам понадобится очень простая лемма.

ЛЕмма 1. Пусть $M$ - полугруппа, связанная с симпличиальнылм конусом C в $\mathbb{R}^{n}$. Тогда

1) если $x_{1}, \ldots, x_{k} \in M$ и $x_{1}+\cdots+x_{k}=0$, то $x_{i}=0$ для любьх $i=1, \ldots, k$;

2) $C+x+y \subset(C+x) \cap(C+y)$ для любых $x, y \in M$.

ДокАзАтЕЛЬСтво. Обозначим через $w_{1}, \ldots, w_{n}$ векторы двойственного базиса для $C$. Так как $\left\langle w_{1}, x\right\rangle \geqslant 0$ для любого $x \in M$, то равенство $x_{1}+\cdots+x_{k}=0$ влечет за собой $\left\langle w_{1}, x_{1}\right\rangle+\cdots+\left\langle w_{1}, x_{k}\right\rangle=0$. Это сумма неотрицательных чисел и, значит, они все равны нулю. Таким образом, $\left\langle w_{1}, x_{1}\right\rangle=\cdots=\left\langle w_{1}, x_{k}\right\rangle=0$ и $x_{i}=0$ для $i=1, \ldots, k$. Это доказывает п. 1). Пусть теперь $z \in C+x+y$. Существует $c \in C$, такой, что $z=x+y+c$. Поскольку $x, y \in M \subset C, z$ принадлежит $(C+x) \cap(C+y)$.

Теорема 2. $S$ - это единственный базис Гильберта полугруппь $M$. 
ДокАЗАтЕЛЬСтво. Пусть $B-$ базис Гильберта полугруппы $M$. Если $x \in S$, то $x$ можно записать в виде

$$
x=n_{1} b_{1}+\cdots+n_{k} b_{k}, \quad n_{i} \in \mathbb{N}, b_{i} \in B .
$$

Таким образом, $C+x \subset C+n_{1} b_{1}$. Множество $C+x$ максимально, потому что $x \in S$, и, таким образом, $x=n_{1} b_{1}$. Если $n_{1}>1$, то

$$
C+x=C+n_{1} b_{1} \subsetneq C+\left(n_{1}-1\right) b_{1} .
$$

Поскольку такого не может быть, $n_{1}=1, x \in B$ и $S \subset B$.

Пусть теперь $b \in B \subset M^{*}, b$ принадлежит некоторому множеству $C+x$, где $x \in S$, и $b=x+c$, где $c \in \mathbb{Z}^{n} \cap C=M$. Таким образом,

И

$$
b=x+n_{1} b_{1}+\cdots+n_{k} b_{k}+m b, \quad n_{i}, m_{i} \in \mathbb{N}, b_{i} \in B, b_{i} \neq b,
$$

$$
x+n_{1} b_{1}+\cdots+n_{k} b_{k}+(m-1) b=0 .
$$

Случай $m \neq 0$ невозможен, поскольку тогда из последнего равенства следовало бы, что $x=0$. Таким образом,

$$
b=x+n_{1} b_{1}+\cdots+n_{k} b_{k} .
$$

Так как $x$ принадлежит $S \subset B$, то $b=x$ и $B=S$.

Определим теперь отношение порядка на $C$. Пусть $x$ и $y-$ два элемента из $C$. Тогда

$$
x \preccurlyeq y \Longleftrightarrow\left\langle w_{i}, x\right\rangle \leqslant\left\langle w_{i}, y\right\rangle \text { для любого } i=1, \ldots, n .
$$

ПРЕДЛОЖЕНИЕ 1 . Если $C-$ симплициальный конус, снабженный отношением порядка $\preccurlyeq$, то

1) $x \preccurlyeq y \Longleftrightarrow C+y \subset C+x$ для любых $x, y \in C$;

2) $S$ - множество минимальных элементов в $M^{*}$.

ДоказАтЕЛЬСтво. Нетрудно проверить, что п. 1) влечет за собой п. 2).

Если $C+y \subset C+x$, то $y \in C+x$; поэтому существует элемент $c \in C$, такой, что $y=c+x$. А поскольку $\left\langle w_{i}, c\right\rangle \geqslant 0$ при $i=1, \ldots, n$, имеем $x \preccurlyeq y$. Обратно, если $x \preccurlyeq y$, положим $c=y-x \in C$. Имеем $y=c+x \in C+x$ и $C+y \subset C+x$.

\section{$\S 2$. Паруса и базисы Гильберта}

Е. И. Коркина приводит в [2] пример рационального симплициального конуса $C$ с таким парусом $V$, что $V \cap \mathbb{Z}^{3}$ не содержит базиса Гильберта для $C \cap \mathbb{Z}^{3}$.

$\mathrm{B} \mathbb{R}^{n}$ имеется простое достаточное условие, при котором $\widetilde{V} \cap \mathbb{Z}^{n}$ является базисом Гильберта для $C \cap \mathbb{Z}^{n}$.

ОПРЕДЕЛЕНИЕ 2. Будем говорить, что многогранник в $\mathbb{R}^{n}$ иелочисленный, если его вершины принадлежат $\mathbb{Z}^{n}$. Будем говорить, что многогранник в $\mathbb{R}^{n}$ nустой, если он является целочисленным и не содержит никаких целых точек, кроме вершин.

ПреДЛОЖение 2. Пусть $C-$ симплициальный конус в $\mathbb{R}^{n}$, a $\widetilde{V}$ u $M-$ соответствуюшие приведенный парус и полугруппа. Если каждая грань паруса $\widetilde{V}$ разбивается на челочисленные симплексы с определителем \pm 1, то $\widetilde{V} \cap \mathbb{Z}^{n}$ является базисом Гильберта полугруппь $M$.

ДоказАтельство. Пусть $x \in C \cap \mathbb{Z}^{n}$ и $x \neq 0$. Прямая, соединяющая начало координат и $x$, пересекает парус $\widetilde{V}$ в точке (см. [4]), которая принадлежит 
некоторой грани этого паруса, а следовательно, и некоторому симплексу $\sigma=$ $\left(O, x_{1}, \ldots, x_{n}\right)$, такому, что $x_{i} \in \mathbb{Z}^{n}$ и $\operatorname{det}\left(x_{1}, \ldots, x_{n}\right)= \pm 1$. Точка $x$, таким образом, принадлежит рациональному симплициальному конусу, порожденному $x_{1}, \ldots, x_{n}$ (координаты точки $x$ в этом базисе неотрицательны).

Пусть $\left(e_{i}\right)$ - стандартный базис в $\mathbb{R}^{n}$, а $H \in \mathrm{GL}_{n}(\mathbb{Z})$ - такой оператор что $H e_{i}=x_{i}$ при $i=1, \ldots, n$. Имеем

$$
H^{-1} x=\sum_{i=1}^{n} a_{i} e_{i}, \quad \text { где } a_{i} \in \mathbb{N},
$$

и $x$ является положительной линейной комбинацией точек $x_{i}$.

В размерности 3 имеется необходимое и достаточное условие. Нам понадобится следуюшая лемма:

ЛЕмма 2. Пусть $a, b, c \in \mathbb{Z}^{3}-$ mакие точки, ито $\operatorname{det}(a, b, c) \neq 0$. Пусть $\sigma$ - симплекс с вериинами $O$ (начало координат), $a, b$ и с. Пусть П - параллелепипед с вершинами $O, a, b, c, b+c, c+a, a+b, a+b+c$ (обозначим П через $P(a, b, c))$. Предположим, что челочисленное расстояние от началс координат до плоскости, содержашей $a, b$ и $c$, равно $d$ и, наконеи, что симплекс $\sigma$ пустой. Обозначим через $v$ линейную рачиональную форму, такую, umo $v(a)=v(b)=v(c)=d$.

$B$ П найдется целая точка $x$, такал, что $v(x)=d+1$. Если $d \geqslant 2$, то можно виббрать $x$ в $\stackrel{\circ}{\Pi}$.

ДокАЗАТЕльство. Целочисленное расстояние от точки $A \in \mathbb{Z}^{n}$ до целочисленной аффинной гиперплоскости $H$ - это отношение евклидова расстояния от $A$ до $H$ к минимальному расстоянию от целой точки (не лежащей в $H$ ) до $H$. Обозначим его через $d(A, H)$. Например, целочисленное расстояние от $(0,0, d)$ до $H=\left\{(x, y, z) \in \mathbb{R}^{3} \mid z=0\right\}$ равно $d$. При вычислении целочисленных расстояний мы всегда будем возвращаться к этой ситуации (что, конечно возможно).

Предположим, что точки $a, b$ и $c$ принадлежат плоскости $z=d$ (в координатах $x, y$ и $z)$. Положим $\Pi^{\prime}=P(O, a, b-a, c-a)$, и пусть $H_{k}$ - плоскости задаваемые уравнениями $z=k$ при $k \in \mathbb{Z}$ (см. рис. 1 ).

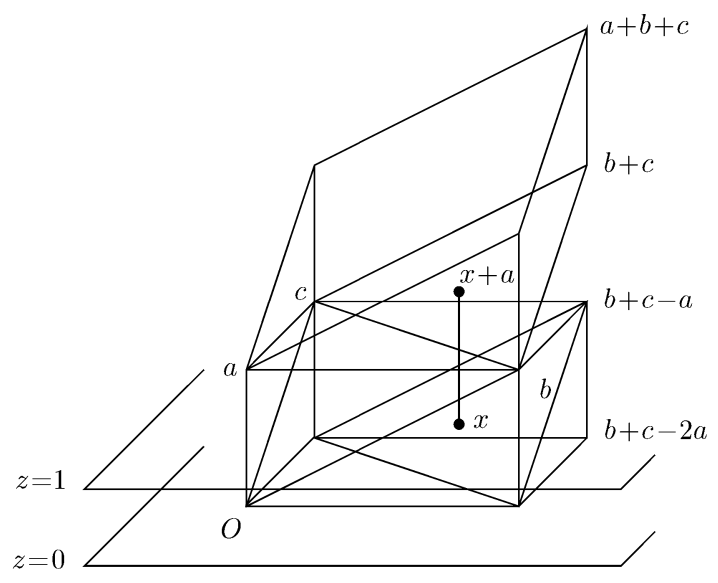

Рис. 1. 
Согласно лемме Минковского, $\Pi^{\prime} \cap H_{k}$ содержит по крайней мере одну целую точку при $0 \leqslant k \leqslant d$.

Пусть $x$ - целая точка в $\Pi^{\prime} \cap H_{1}$, и рассмотрим симплекс $\sigma^{\prime}=-\sigma+b+c-a$. Этот симплекс содержится в $\Pi^{\prime}$ и является пустым, поскольку $\sigma$ пустой. Мы видим, таким образом, что $x$ не принадлежит внутренности симплекса $\sigma$ или $\sigma^{\prime}$ (но $x$ может быть вершиной одного из этих симплексов). Отсюда вытекает, что $x+a \in \Pi$, и так как $v(x)=1$, то $v(a+x)=d+1$.

Обратимся теперь к последнему утверждению и рассмотрим октаэдр $\Omega$ с вершинами $O, b, c, b-a, c-a, b+c-a$. Грани этого октаэдра параллельны граням симплексов $\sigma$ и $\sigma^{\prime}$. Целая точка в $\Omega$ должна либо быть вершиной, либо лежать в $\stackrel{\circ}{\Omega}$. Заметим, что вершины октаэдра $\Omega$ лежат на плоскостях $H_{0}$ и $H_{d}$. Таким образом, если $d \geqslant 2$, то точка $x$, выбранная в $\Pi \cap H_{1}$, принадлежит $\Omega$ и $x+a \in \stackrel{\circ}{\Pi}$.

Теорема 3. Пусть $C$ - симпличиальньй конус в $\mathbb{R}^{3}$, a $\widetilde{V}$ и $M-$ соответствующие приведенньй парус и полугруппа. Следующие свойства эквивалентнь:

1) $\widetilde{V} \cap \mathbb{Z}^{3}$ является базисом Гильберта для $M$;

2) челочисленное расстояние между гранями паруса $\widetilde{V}$ и началом координат равно 1 .

ДокАЗАтЕЛЬСтво. Пусть $H$ - целочисленная плоскость, такая, что $d(O, H)$ $=1$, и пусть $x_{1}, x_{2}$ и $x_{3}$ - три целые точки в $H$, такие, что $\operatorname{det}\left(x_{1}, x_{2}, x_{3}\right) \neq 0$. Если треугольник $\left(x_{1}, x_{2}, x_{3}\right)$ пустой, нетрудно проверить, что $\operatorname{det}\left(x_{1}, x_{2}, x_{3}\right)$ $= \pm 1$.

Предположим, что целочисленное расстояние от граней паруса $\widetilde{V}$ до начала координат равно 1 . Если $F-$ грань паруса $\widetilde{V}$, то она представляет собой компактный многоугольник с целыми вершинами, лежащий на аффинной целочисленной плоскости $H$. Разобьем $F$ на пустые треугольники. Предыдущее замечание показывает, что мы можем применить предложение 2 и заключить, что $\widetilde{V} \cap \mathbb{Z}^{3}$ является базисом Гильберта для $M$.

Теперь предположим, что приведенный парус $\widetilde{V}$ содержит грань $F$, такую, что $d(O, F)=d \geqslant 2$. Пусть $(a, b, c)$ - пустой треугольник, содержащийся в $F$. Симплекс $(O, a, b, c)$ пуст. Пусть теперь $v$ - линейная форма, такая, что $v(a)=$ $v(b)=v(c)=d$. Во внутренности параллелепипеда $P(a, b, c)$ существует такая целая точка $x$, что $v(x)=d+1(x$ не принадлежит $V)$. Для всякой точки $z \in V$ имеем $v(z) \geqslant d$, так что если $x$ является целочисленной линейной комбинацией точек из $V \cap \mathbb{Z}^{3}$,

$$
x=n_{1} u_{1}+\cdots+n_{k} u_{k}, \quad \text { где } n_{i} \in \mathbb{N}, u_{i} \in V \cap \mathbb{Z}^{3},
$$

TO

$$
v(x)=d+1 \geqslant\left(n_{1}+\cdots+n_{k}\right) d .
$$

Но неравенство $d \geqslant 2$ влечет за собой равенство $n_{1}+\cdots+n_{k}=1$, и все $n_{i}$ равны 0, кроме одного, равного 1. Мы получили противоречие, которое показывает, что $\widetilde{V} \cap \mathbb{Z}^{3}$ не является базисом Гильберта для $M$. 


\section{$\S 3$. Примеры}

Мы вычислили несколько рациональных трехмерных парусов, но ни один из них не удовлетворяет условиям теоремы 3. Тем не менее такие паруса существуют. Простейший из них - парус симплициального конуса, порожденного точками $(1,0,0),(0,1,0)$ и $(0,0,1)$.

Можно получить более сложный пример следующим образом. Положим $a_{1}=$ $(1,0,0), a_{2}=(0,1,0)$ и $a_{3}=(0,0,1)$. Эти три точки определяют симплициальный конус, который можно расширить. Ищем точку $a_{4}$, такую, что

(1) $\operatorname{det}\left(a_{1}, a_{2}, a_{4}\right)= \pm 1$;

(2) $\operatorname{det}\left(a_{1}, a_{3}, a_{4}\right)= \pm 1$;

(3) грани приведенного паруса симплициального конуса, порожденного точками $a_{2}, a_{3}$ и $a_{4}$, 一 треугольники $\left(a_{1}, a_{2}, a_{3}\right),\left(a_{1}, a_{2}, a_{4}\right)$ и $\left(a_{1}, a_{3}, a_{4}\right)$.

Если $a_{4}=(x, y, z)$, то из первого условия вытекает, что $z= \pm 1$, а из второго 一 что $y= \pm 1$. Условие (3) говорит о том, что $O$ отделена от $a_{4}$ плоскостью $\left(a_{1}, a_{2}, a_{3}\right)$, от $a_{2}$ - плоскостью $\left(a_{1}, a_{3}, a_{4}\right)$ и от $a_{3}$ - плоскостью $\left(a_{1}, a_{2}, a_{4}\right)$. Из этих условий вытекают неравенства $x+y+z>1, y(x+y+z-1)<0$ и $z(x+y+z-1)<0$. Таким образом, $y$ и $z$ должны быть отрицательными числами. Следовательно, $y=z=-1$. Наконец, можно выбрать $x=4: a_{4}=(4,-1,-1)$.

Мы получили рациональный приведенный парус с тремя гранями, такими, что целочисленное расстояние от каждой грани до начала координат равно 1. Мы можем сделать еще один шаг вперед и попытаться найти точку $a_{5}=$ $(x, y, z)$, такую, что

(1) $\operatorname{det}\left(a_{2}, a_{4}, a_{5}\right)= \pm 1$;

(2) $\operatorname{det}\left(a_{2}, a_{3}, a_{5}\right)= \pm 1$;

(3) грани приведенного паруса симплициального конуса, порожденного точками $a_{3}, a_{4}$ и $a_{5}$, 一 треугольники $\left(a_{1}, a_{2}, a_{3}\right),\left(a_{1}, a_{2}, a_{4}\right),\left(a_{1}, a_{3}, a_{4}\right)$, $\left(a_{2}, a_{4}, a_{5}\right)$ и $\left(a_{2}, a_{3}, a_{5}\right)$.

Из этих условий получаем $4 z+x= \pm 1$ и $x= \pm 1$. Принимая во внимание условие $(3)$, выбираем $a_{5}=(-1,3,0)$. Можно проверить, что парус симплициального конуса, порожденного $a_{3}, a_{4}$ и $a_{5}$ - в точности то, что нам нужно. Мы можем продолжить наши построения, но только слегка видоизменив конструкцию. Первые два уравнения, которые мы написали для нахождения точек $a_{4}$ и $a_{5}$, определяют две плоскости. Пересечение соответствующих плоскостей, определяемых для нахождения точки $a_{6}$, не содержит ни одной целой точки.

Мы построим плоскость $R$, такую, что

(1) $R$ содержит $a_{3}$ и $a_{4}$;

(2) $d(O, R)=1$;

(3) $O$ отделена от $a_{i}$ при $i=1, \ldots, 5$ плоскостью $R$,

и плоскость $L$, такую, что

(1) $L$ содержит $a_{3}$ и $a_{5}$;

(2) $d(O, R)=1$;

(3) $O$ отделена от $a_{i}$ при $i=1, \ldots, 5$ плоскостью $L$. 
Прямая $L \cap R$ является целочисленной и содержит целую точку $a_{3}$. Выберем точку $a_{6}$ на этой прямой, учитывая то, что $O$ и $a_{6}$ должны быть отделены друг от друга плоскостью $\left(a_{3}, a_{4}, a_{5}\right)$. Повторяя описанные шаги, можно получить рациональный парус со сколь угодно большим числом граней.

Мы должны отметить, что теорема 3 не выполняется в размерности $n \geqslant 4$. В самом деле, пусть $\sigma$ - целочисленный симплекс в $\mathbb{R}^{4}$ с вершинами $O, a_{1}=$ $(0,0,0,1), a_{2}=(1,0,0,1), a_{3}=(0,1,0,1)$ и $a_{4}=(1,1,-d, 1)$, где $d \in \mathbb{N}^{*}$. Пусть $C, V$ и $M$ - соответствующие симплициальный конус, парус и полугруппа. Заметим, что точки $a_{i}$ лежат на гиперплоскости $t=1$ (в координатах $x, y, z, t$ ), что $\operatorname{det}\left(a_{1}, a_{2}, a_{3}, a_{4}\right)=d$ и что в гиперплоскости $t=1$ симплекс $\left(a_{1}, a_{2}, a_{3}, a_{4}\right)$ пустой. Таким образом, $V \cap \mathbb{Z}^{4}$ не является базисом Гильберта для $M$.

Мне приятно поблагодарить В. И. Арнольда, который терпеливо следил за работой, и Е. И. Коркину за помощь, особенно с примерами.

\section{ЛИТЕРАТУРА}

1. Arnold V. I. A-graded algebras and continued fractions. Commun. Pure Appl. Math., 42, 993-1000 (1989).

2. Korkina E. Classification of A-graded algebas with 3 generators. Indag. Math. (N.S.), 3 (1), 27-40 (1992).

3. Коркина Е. И. Двумерные непрерывные дроби. Простейшие примеры. Труды МИАН, 209, 143-166 (1995).

4. Lachaud G. Voiles et Polyédres de Klein. Prétirage 95-22, Laboratoire de Mathématiques Discrétes, C.N.R.S., 1995.

CEREMADE, University Paris 9

e-mail: msfr@pi.ceremade.dauphine.fr
Поступило в редакцию 16 сентября 1998 г. 